\title{
Vapor Pressures and PVT Properties of HFE-245mc (pentafluoroethyl methyl ether)
}

\author{
Toshihide Tsuge, Haruki Sato, and Koichi Watanabe \\ Department of System Design Engineering, Faculty of Science and Technology, Keio University \\ 3-14-1, Hiyoshi, Kohoku-ku, Yokohama 223, Japan
}

\begin{abstract}
Since HFE-245mc has both lower ozone depletion potential (ODP) and smaller global warming potential (GWP), it is expected being a possible candidate to replace R-114. We report a complete set of vapor pressures, PVT properties of HFE$245 \mathrm{mc}$ measured by a constant volume method. One hundred and ten vapor pressures were obtained from $310 \mathrm{~K}$ to the critical temperature $(406.80 \mathrm{~K})$ and a vapor pressure correlation has been developed. One hundred and twenty-four $P V T$ property data in the range of temperatures from $360 \mathrm{~K}$ to $440 \mathrm{~K}$ and pressures up to $8.4 \mathrm{MPa}$ were also obtained along 12 isochores. A virial-expansion type equation of state to represent the present measurements has also been developed.

[alternative refrigerant, vapor pressures, PVT properties, constant volume method, equation of state]
\end{abstract}

\section{Introduction}

In accord with an increasing concern about the global warming issue, even HFC (hydrofluorocarbon) alternative refrigerants to replace conventional HCFC (hydrochlorofluorocarbon) refrigerants are currently not considered as a long-term substitute, as a working fluid for air-conditioning, heat-pumping and refrigerating equipments throughout the forthcoming 21st century, although HFCs virtually have no potential to deplete the stratospheric ozone layer.

As one of our national R\&D projects, therefore, the New Energy and Industrial Technology Development Organization (NEDO) has devoted, for the last several years, to search socalled "new generation refrigerants" which should have far lower GWP than HFCs. Among the candidates thus proposed recently [1] by the Research Institute of Innovative Technology for the Earth (RITE), there exists HFE-245mc (pentafluoroethyl methyl ether) as a promising candidate to replace R-114 especially for vapor-compression heat pump system applications.

The present study aims to provide reliable thermodynamic property data on HFE- $245 \mathrm{mc}$ and thermodynamic model to represent them so as to challenge such an application mentioned above.

\section{Experimental}

In the present study, a constant volume apparatus shown in Fig. 1 has been used to measure vapor pressures and PVT properties. This apparatus is similar to that used in our earlier studies for R-134a [2], R-123 [3], R-125 [4] and R-236ea [5] and reliability has been confirmed. A prescribed mass of the sample with research grade purity $(99.9967 \mathrm{~mol} \%)$ is confined into a spherical thick walled sample-cell (A) made of stainless steel with an inner volume of about $139 \mathrm{~cm}^{3}$ and installed in a thermostated fluid-bath (C).
The sample temperature is not detected directly but is considered being the same as that of heat transfer medium within the thermostated fluid bath, since a series of measurements have been performed under a well-established thermal equilibrium condition between the sample fluid and bath fluid. The temperature of bath fluid is automatically controlled within \pm 2 $\mathrm{mK}$ of its fluctuation using a PID controller $(\mathrm{O})$, detected by a platinum resistance thermometer $(\mathrm{T})$, and measured with a thermometer bridge (B).

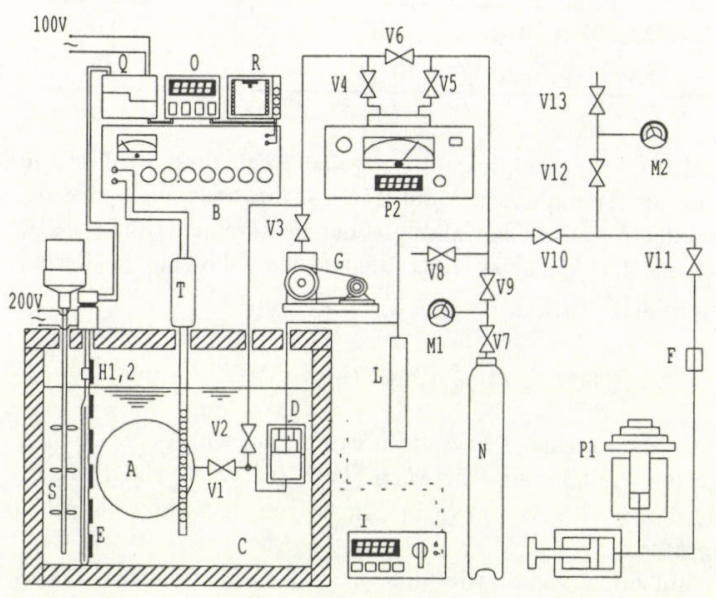

Fig. 1. Experimental apparatus

[A] : Sample cell [B] : Thermometer bridge [C]: Thermostated bath [D]: Differential pressure indicator $[\mathrm{E}]$ : Partition plate $[\mathrm{F}]$ : Dust filter $[\mathrm{G}]$ : Vacuum pump $[\mathrm{H} 1,2]$ : Main / Sub heater $[\mathrm{I}]$ : Digital resistance meter [J] : Pressure damper $[\mathrm{M} 1,2]$ : Pressure gauges $[\mathrm{N}]$ : Nitrogen bottle [O] : PID controller [P1] : Air piston type pressure gauge [P2] : Bourdon-tube pressure gauge $[\mathrm{Q}]$ : Thyristor regulator $[\mathrm{R}]$ : Pen recorder $[\mathrm{S}]$ : Stirrer $[\mathrm{T}]$ : Platinum resistance thermometer [V1-13]: Valves 
The sample pressure is then measured, with the aid of an air piston pressure gauge (P1), as that of nitrogen gas [supplied from its bottle $(\mathrm{N})$ ] which serves as a pressure transferring medium existing at the upper space of the stainless-steel membrane equipped in a differential pressure indicator (D).

The sample density, on the other hand, is determined on the basis of the sample mass weighed by a precision chemical balance within the estimated uncertainty of $\pm 3 \mathrm{mg}$ and the inner volume of the sample cell associated with required minor corrections owing to thermal expansion and hydrostatic pressure deformation.

The experimental uncertainties of the present measurements have been estimated to be not greater than $\pm 10 \mathrm{mK}$ in temperature, $\pm 1.4 \mathrm{kPa}$ in pressure and $\pm 0.07 \%$ in density, respectively.

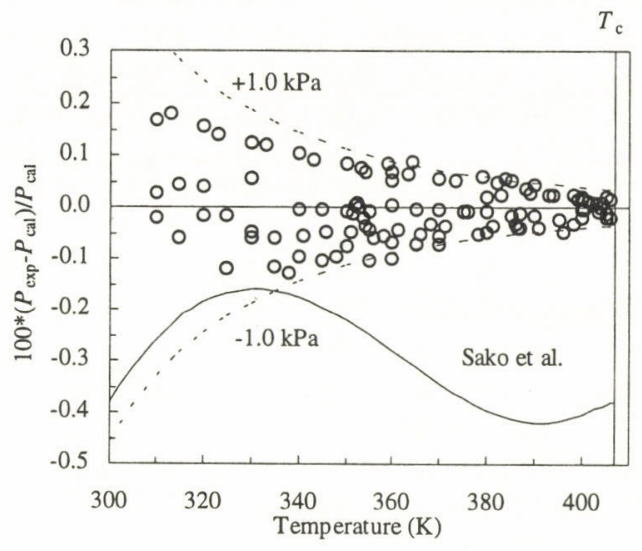

Fig. 2. Vapor pressure deviations of HFE-245mc from eq. (1)

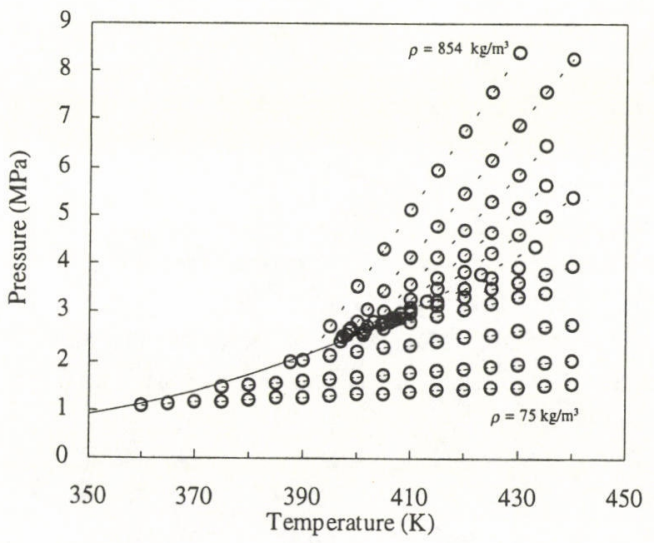

Fig. 3. Distribution of experimental $P V T$ data

\section{Results and Discussion}

3.1 Vapor pressures

One hundred and ten vapor pressures have been obtained for temperatures from $310 \mathrm{~K}$ to the critical temperature, which correspond to pressures between 0.3 $\mathrm{MPa}$ and $2.9 \mathrm{MPa}$. Based on the obtained data, a three-coefficient Wagner-type vapor pressure correlation has been developed as:

$$
\ln P_{\mathrm{r}}=\frac{1}{T_{\mathrm{r}}}\left(a_{1} \tau+a_{2} \tau^{1.4}+a_{3} \tau^{3.0}\right)
$$

where $P_{\mathrm{r}}=P_{\mathrm{s}} / P_{\mathrm{c}}$, is the reduced vapor pressure of HFE-245mc, $P_{\mathrm{s}}$ and $P_{\mathrm{c}}$ are the vapor pressure and the critical pressure of HFE-245mc, respectively, $T_{\mathrm{r}}=T / T_{\mathrm{c}}$, is the reduced temperature and $\tau$ is defined as $\tau=1-T_{\mathrm{r}}$. The numerical constants in eq. (1) are $a_{1}=-7.8491086, a_{2}=1.4699487$, and $a_{3}=-3.6933492$. The critical pressure $P_{\mathrm{c}}$ was also determind as a fitting parameter of eq. (1) as well as $a_{1}$ to $a_{3}$ :

$$
P_{\mathrm{c}}=2886.3 \pm 1.5 \mathrm{kPa}
$$

which corresponds to $T_{\mathrm{c}}=406.80 \pm 0.03 \mathrm{~K}$ reported by Sako et al [6]. This critical pressure agrees with reference [6] within the reported uncertainty.

The average deviation between the present measurements and eq. (1) is $0.05 \%$, while the maximum deviation is $0.18 \%$. Figure 2 shows the comparison of the present measured data and vapor pressure correlation reported by Sako et al. [7] with eq. (1). Most of the present measurements are well represented within the absolute deviation of $\pm 1.0 \mathrm{kPa}$ by eq. (1). Vapor pressures calculated from the correlation proposed by Sako et al. are lower than eq. (1) for almost the entire range of temperatures and absolute deviation amounts to $-11.0 \mathrm{kPa}$ at $406.74 \mathrm{~K}$, the critical temperature reported earlier by Sako et al. [7].

Table 1. Numerical coefficients in eq. (3)

\begin{tabular}{rcrr}
\hline$i$ & $a_{i}$ & $\alpha_{i}$ & $\beta_{i}$ \\
\hline 1 & -1.8114918 & & \\
2 & 1.8596257 & 0 & 2 \\
3 & -2.5992019 & -1 & 2 \\
4 & 3.8160201 & -1 & 3 \\
5 & 9.8899218 & -2 & 3 \\
6 & -9.0506900 & -3 & 3 \\
7 & $-2.4845810 \times 10^{1}$ & -2 & 4 \\
8 & $1.9302835 \times 10^{1}$ & -3 & 4 \\
9 & 8.2997099 & -1 & 5 \\
10 & -7.0732112 & -2 & 6 \\
11 & -1.2449543 & -1 & 7 \\
12 & 5.9689394 & -2 & 7 \\
13 & -1.8778413 & -3 & 7 \\
14 & $-4.1830091 \times 10^{-1}$ & -1 & 8 \\
\hline \multicolumn{5}{c}{$P_{\mathrm{c}}=2886.3 \mathrm{kPa}$} & $T_{\mathrm{c}}=406.80 \mathrm{~K}$ \\
$\rho_{\mathrm{c}}=$ & $499 \mathrm{~kg} / \mathrm{m}^{3}$ & $M=150.054$ \\
$R_{\mathrm{o}}=8.31451 \mathrm{~kJ} /(\mathrm{kmol} \cdot \mathrm{K})$ & \\
\hline
\end{tabular}



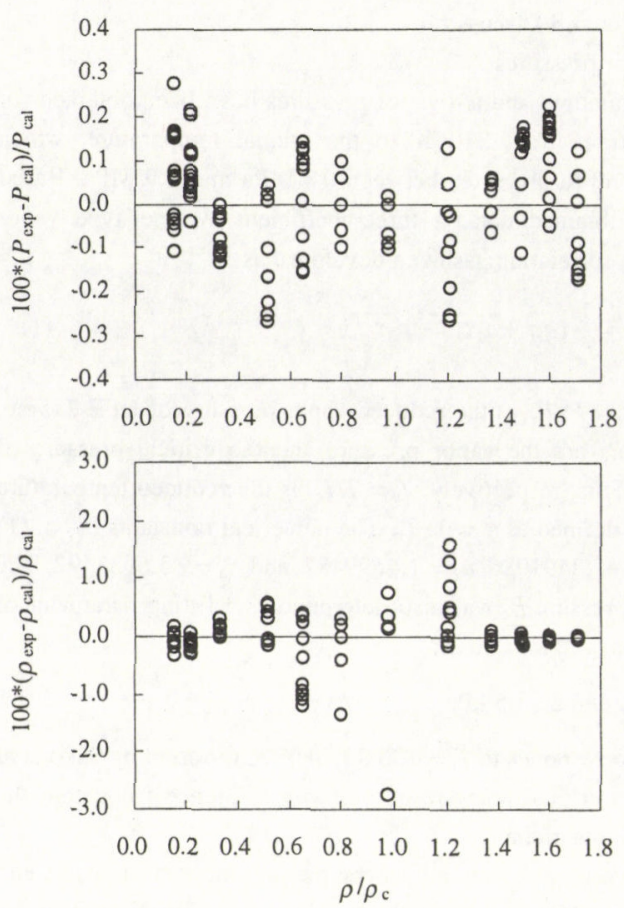

Fig. 4. Pressure and density deviations of experimental PVT data from eq. (3)

\subsection{PVT Properties}

A total of $124 P V T$ properties of HFE- $245 \mathrm{mc}$ have been measured along 12 different isochores covering an extensive range of temperatures $360-440 \mathrm{~K}$, pressures 1.1-8.4 MPa and densities $75-854 \mathrm{~kg} / \mathrm{m}^{3}$ in the present study. A distribution of the present measurements is illustrated on a pressure-temperature diagram with our vapor pressure curve in Fig. 3.

All the $P V T$ data have been used to fit a 14-coefficient virialexpansion type equation of state given below:

$$
P_{\mathrm{r}}=\frac{\rho_{\mathrm{r}} T_{\mathrm{r}}}{Z_{\mathrm{c}}}+a_{1} \exp \left(\frac{1}{T_{r}}\right) \rho_{\mathrm{r}}{ }^{2}+\sum_{i=2}^{14} a_{i} \rho_{\mathrm{r}}^{\beta_{i}} T_{\mathrm{r}}^{\alpha_{i}}
$$

where, $P_{\mathrm{r}}=P / P_{\mathrm{c}}, T_{\mathrm{r}}=T / T_{\mathrm{c}}, \rho_{\mathrm{r}}=\rho / \rho_{\mathrm{c}}, Z_{\mathrm{c}}=P_{\mathrm{d}} /\left(\rho_{\mathrm{c}} R T_{\mathrm{c}}\right), R=R_{\mathrm{d}} / M$, and $R_{0}$ and $M$ are the universal gas constant and molar mass of HFE$245 \mathrm{mc}$, respectively. The values of all the parameters and numerical constants in eq. (3) are listed in Table 1. Pressure and density deviations of experimental PVT data from eq. (3) are shown in Fig.4 for different reduced densities. This equation represents satisfactorily the present experimental data within $\pm 0.3 \%$ in pressure and within $\pm 0.5 \%$ in density deviation for most of the present measurements, respectively. This equation of state is effective for the range of temperatures $350-450 \mathrm{~K}$, pressures up to $9.0 \mathrm{MPa}$, and densities up to $900 \mathrm{~kg} / \mathrm{m}^{3}$.
We have then examined the thermodynamic behavior of the isobaric specific heat capacities of HFE-245mc along isobars on the basis of the developed model and we found it quite reasonable. However, some minor modifications were found necessary to be improved with respect to the isochoric heat capacity behavior especially in the liquid phase.

\section{Conclusion}

In this study, an extensive set of vapor pressures and PVT properties of HFE-245mc has been measured. A vapor pressure correlation has been developed, and the critical pressure $P_{\mathrm{c}}=$ $2886.3 \pm 1.5 \mathrm{kPa}$ which corresponds to the critical temperature $T_{\mathrm{c}}=406.80 \pm 0.03 \mathrm{~K}$ has also been determined. The present experimental PVT measurements are the first set of data that cover entire fluid phase and these measurements have been represented by the virial-expansion type equation of state. This equation of state is the first ever formulated based on the experimental data for HFE-245mc. In addition, however, it seems essential to the present authors that further accumulation of reliable thermodynamic property data including speeds of sound, and isobaric specific heat capacities, etc. is urgently needed. Heat capacity values in the ideal gas state are also essential to be studied in the nearest future.

\section{Acknowledgement}

This research was partially supported by NEDO through RITE, and the authors are grateful to these organizations. We also wish to thank Akira Nakashima, a graduate student at Keio University, and Atsuko Uchimura, an undergraduate student at Keio University, for their assistance.

\section{References}

[1] S. Misaki and A. Sekiya, Proc. of Int. Conf. on Ozone Protection Technologies, Washington, D. C., pp 65-70 (1996).

[2] C.-C. Piao, H. Sato, and K. Watanabe, ASHRAE Trans., 96, pp 132-140 (1990).

[3] C.-C. Piao, H. Sato, and K. Watanabe, J. Chem. Eng. Data, 36, pp 398-403 (1991).

[4] T. Sagawa, H. Sato, and K. Watanabe, High Temp.-High Press., 26, pp 193-201 (1994).

[5] S. Kuroda, H. Sato, and K. Watanabe, Proc. of 1995 JAR Annual Conf., Tokyo, pp 117-120, (1995).

[6] T. Sako, M. Sato, N. Nakazawa, M. Oowa, M. Yasumoto, H. Ito, and S. Yamashita, J. Chem. Eng. Data, 41 (4), pp 802805 (1996).

[7] T. Sako, M. Sato, N. Nakazawa, M. Oowa, A. Sekiya, H. Ito, and S. Yamashita, Proc. Joint Meeting of IIR Commissions, CFCs The Day After, Padova, pp 485-491 (1994). 